\title{
Juno observations of spot structures and a split tail in Io- induced aurorae on Jupiter
}

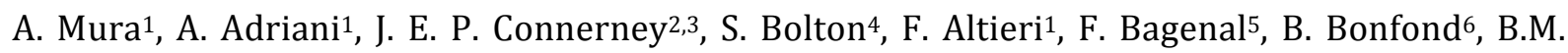 \\ Dinelli $^{7}$, J-C. Gerard6, T. Greathouse ${ }^{4}$, D. Grodent 6 , S. Levin', B. Mauk ${ }^{9}$, M.L. Moriconi' ${ }^{7}$, J. Saur ${ }^{10}$, J. \\ H. Waite Jr.4,11, M. Amoroso ${ }^{12}$, A. Cicchetti ${ }^{1}$, F. Fabiano ${ }^{7}$, G. Filacchione ${ }^{1}$, D. Grassi ${ }^{1}$, A. Migliorini' ${ }^{1}$, \\ R. Noschese ${ }^{1}$, A. Olivieri ${ }^{12}$, G. Piccioni ${ }^{1}$, C. Plainaki ${ }^{1,12}$, G. Sindoni ${ }^{12}$, R. Sordini ${ }^{1}$, F. Tosi ${ }^{1}$, D. Turrini ${ }^{1}$ \\ ${ }^{1}$ Institute for Space Astrophysics and Planetology, National Institute for Astrophysics, Italy. \\ 2Space Research Corporation, Annapolis, MD, USA. \\ ${ }^{3}$ NASA Goddard Space Flight Center, USA. \\ ${ }^{4}$ Southwest Research Institute, USA. \\ ${ }^{5}$ University of Colorado Boulder, USA. \\ ${ }^{6}$ Space Science, Technologies and Astrophysical Research Institute, Laboratory for Planetary and \\ Atmospheric Physics, University of Liège, Belgium. \\ ${ }^{7}$ Institute of Atmospheric Sciences and Climate, National Research Council, Italy. \\ ${ }^{8}$ Jet Propulsion Laboratory, California Institute of Technology, USA. \\ ${ }^{9} J o h n s$ Hopkins University Applied Physics Laboratory, USA. \\ ${ }^{10}$ University of Cologne, Germany. ${ }^{11}$ University of Texas, San Antonio, USA. ${ }^{12}$ Agenzia Spaziale Italiana, Italy.
}

\begin{abstract}
Jupiter's aurorae are produced in its upper atmosphere when incoming high-energy electrons precipitate along the planet's magnetic field lines. A northern and a southern main auroral oval are visible, surrounded by small emission features associated with the Galilean moons. We present infrared observations, obtained with the Juno spacecraft, showing that in the case of Io, this emission exhibits a swirling pattern that is similar in appearance to a von Kármán vortex street. Well downstream of the main auroral spots the extended tail is split in two. Both of Ganymede's footprints also appear as a pair of emission features, which may provide a remote measure of Ganymede's magnetosphere. These features suggest that magnetohydrodynamic interaction between Jupiter and its moon is more complex than previously anticipated.
\end{abstract}

Like the Earth, Jupiter has aurorae. These permanent electro-magnetic emissions are associated with the precipitation of magnetospheric plasma onto the planet's ionosphere. Jupiter's aurorae have emission features, absent at Earth, associated with its moons: bright spots appear in the ionosphere at the base of the magnetic field lines that sweep past the Galilean moons Io, Europa, and Ganymede. The discovery of auroral features at the magnetic field footprints of Io $(1,2)$ showed the electromagnetic interaction between Jupiter and its moons. In situ observations by the Galileo spacecraft of intense bi-directional, magnetic field-aligned electron beams, with energies up to $200 \mathrm{keV}$, within the wake and over the poles of Io showed that these interactions can lead to strong electron acceleration (3-5).

Infrared and ultraviolet observations of Jupiter's auroral footprints led to theoretical understanding that the equatorial magnetospheric plasma roughly corotates with the planet, but the moons, which orbit more slowly, form an obstacle to this plasma $(6,7)$. This interaction leads to the formation of magnetohydrodynamic waves, specifically Alfvén waves $(7,8)$, which accelerate electrons toward the planet's atmosphere, where they excite auroral emissions (9). While electrons travel rapidly along the magnetic field lines, the slower Alfvén waves are convected downstream, resulting in a standing Alfvén wing that is slightly tilted away from the undisturbed magnetic field line (8). The speeds of the waves are highly variable, depending on the path toward Jupiter through different plasma environments (10). Waves generated at Io first travel a dense plasma torus around the moon, and then into the low-density magnetosphere lobe regions (Fig. 1). Successive traversals of plasma discontinuities may lead to multiply reflected Alfvén waves, which has been suggested as a possible cause of the observed multiple footprint features (11-13).

The observation of auroral $\mathrm{H}_{3}{ }^{+}$emission in relation to the positions of the moons, where the interaction 
occurred, is a powerful tool for remote diagnostic of the Jovian magnetosphere (9).

The Jovian Infrared Auroral Mapper (JIRAM) (14) on NASA's Juno spacecraft $(15,16)$ has been observing the Jovian aurora since 2016 (17) from a polar, highly elliptical orbit. From its position above the poles, JIRAM has obtained views of Jupiter's auroral regions (18). JIRAM is an infrared (IR) imager and spectrometer; one of the imaging channels operates in the range 3.3-3.6 $\mu \mathrm{m}$, where some of the most intense $\mathrm{H}_{3}{ }^{+}$auroral emission lines are observed. During Juno's 8th close pass of the planet (1 September 2017), JIRAM targeted the predicted location of the Io footprint (IFP).

In Fig. 2 we show the IFP peak emissions observed by JIRAM on that occasion at a spatial resolution of $\sim 20 \mathrm{~km}$. Io orbits Jupiter rapidly, so its longitude in the reference frame of the planet was $\sim 80^{\circ} \mathrm{W}$ during the northern observations and $\sim 135^{\circ} \mathrm{W}$ during the southern observations. In both the northern and southern hemispheres, the main IFP is preceded by a $\sim 1000 \mathrm{~km}$ long, thin emission feature, interpreted as the IR signature of electron beams accelerated in both hemispheres (TEB, trans-hemispheric electron beam) (13). However, on this occasion, the precursor feature is not detached from the rest of the footprint. The characteristic decay time of $\mathrm{H}_{3}{ }^{+}$is a few hundred seconds, (19) longer than the almost instantaneous ultraviolet (UV) hydrogen-related time-scale (12), which may explain the blurring and extent of the TEB signature in the IR, unlike previous UV observations (13).

Figure 2A shows that the southern main spot is followed by a series of regularly spaced secondary spots, which are alternately displaced poleward and equatorward of the median track. The transverse displacement is $100 \pm 10 \mathrm{~km}$. By tracing back to the orbit of Io using a magnetic field model (20), this distance corresponds to $4000 \pm 1000 \mathrm{~km}$, roughly the size of Io itself. The main spot location is exactly along the track predicted by the model (20), and the repetition step of the features is $350 \pm 10 \mathrm{~km}$.

Given the relative velocity of the main spot over the surface of Jupiter, the separation of secondary spots corresponds to approximately $60 \mathrm{~s}$. The chemical lifetime of $\mathrm{H}_{3}{ }^{+}$is longer (up to $1000 \mathrm{~s}$ ) (19) and it is expected to produce extensive smearing, which should hide the observed small-scale structure of the spots, as proposed for the leading TEB spot. A possible explanation is that the secondary spots are generated by higher energy electrons. A model of inertial Alfvén waves acceleration (21) indicates that the electron energy spectrum might be different for the direct, main Alfvén wing and the conjugate (TEB) electron beams. High-energy electrons would precipitate deeper into the atmosphere, producing $\mathrm{H}_{3}+$ ions where their lifetime is shorter due to the high methane density $\mathrm{CH}_{3}{ }^{+}$destruction occurs via the reaction $\mathrm{H}_{3}{ }^{+}+\mathrm{CH}_{4} \rightarrow \mathrm{CH}_{5}{ }^{+}+\mathrm{H}_{2}$ ) (22). An alternative explanation, for the blurring of the precursor feature, could be that the electron beam that creates the TEB spot, formed by up-going electrons from the opposite hemisphere, broadened on its way along the field line through the whole magnetosphere.

The 60 s separation between spots, and the similar intensities of subsequent spots, are difficult to explain with multiple specular reflections, neither inside nor outside the dense plasma torus (10). However, highamplitude nonlinear waves are generally not reflected specularly. The multiplicity and shape of the spots can be explained by models (23), in which strong non-linearities trigger wave interference patterns leading to the occurrence of multiple spots, depending on Io's location in the torus. The observations suggest that the Alfvén waves might be broken up into smaller waves with different travel paths leading to a turbulent emission pattern. The angular offset of the elongated main spot and the alternating displacements of the tail spots might be influenced by the Hall effect in Io's ionosphere, expected to rotate the Alfvén waves (24). Alternatively, since this feature superficially resembles a von Kármán vortex street (a hydrodynamically-observed phenomena) (25), this may result from Io-produced flow shears that generate vortices downstream in the plasma-atmosphere interaction region.

In the northern hemisphere (Fig. 2, C to E) the shape of the Io footprint is generally consistent with that observed in the south: an array of secondary spots is visible in the first part of the tail. Away from the main IFP, the tail becomes gradually more turbulent, likely due to a continuation of the Alfvénic interaction, similar to the main spot (26). 
Each spot is about $200 \mathrm{~km}$ wide. Because JIRAM was observing from angle of $\sim 35^{\circ}$ between the vertical direction and the line of sight, this means that the height of the main $\mathrm{H}_{3}{ }^{+}$emission region cannot be taller than $200 \mathrm{~km} / \tan \left(35^{\circ}\right) \approx 300 \mathrm{~km}$. This short column height is consistent with generation by high-energy electrons (27).

Extrapolating the radiance collected by JIRAM filter to the full $\mathrm{H}_{3}{ }^{+}$spectrum, and integrating it over the surface, the total radiated energy can be estimated at $\sim 6 \mathrm{GW}$ in the south and $\sim 3 \mathrm{GW}$ in the north, $\sim 3$ times less than in the UV (28). The north-south asymmetry of the emission, both in shape but especially in intensity, is a recurrent feature of Jupiter's aurorae (30). Some hypotheses have been proposed (31) to explain this as a consequence of the north-south magnetic field asymmetries: the magnetic field controls the electron acceleration efficiency and the probability for an electron to reach the atmosphere. In our case, the difference may also be due to differing observation times.

Figure 3 shows the far tail of Io footprint as observed on 27 August 2016 in the northern hemisphere. The main spot is $\sim 100^{\circ}$ upstream in longitude from this region. In this sector, we find the tail consists of two separate parallel arcs. The poleward arc is thicker, more intense and more turbulent, while the equatorward arc is fainter, thinner, and less turbulent. These features are visible in previous and subsequent images (taken within one hour), and extend for at least $70^{\circ}$ along the tail. This double footprint tail has not been observed in UV images, probably due to insufficient spatial resolution. However, other JIRAM images with similar spatial resolution [(18), their Figs. 2 and 3] show a single tail, indicating that the double feature is not persistent or not present at all longitudes.

Compared to the cross-track displacement of the IFP tail close to Io shown in Fig. $2(\sim 100 \mathrm{~km})$, the separation in Fig. 3 is larger: the parallel tail components are separated by 500 to $1000 \mathrm{~km}$. The viewing angle in Fig. 3 is less than $10^{\circ}$, ruling out the possibility that JIRAM was observing a vertical (rather than horizontal) structure. The difference in the shape and intensity of the two arcs could be due to the Hall effect generating brighter aurorae in Io's atmosphere on the anti-Jovian side than on the Jovian side (24). The poleward part of the corresponding footprint on Jupiter should then be brighter than the equatorward part, as observed. We suggest that the smooth tail might arises from trans-hemispheric electrons similarly to the TEB (13) feature in Fig. 2A, while the other tail might be related to the wave, which would produce a patchier tail. The splitting could then be due to a northern anomaly in Jupiter's magnetic field (33), which might also explain why this bifurcation is not observed in the southern aurora.

The comparison of the IFP with the footprint of Ganymede (GFP) can provide insight into the mechanisms that generate these morphologies. Figure 4 shows several images of the GFP (taken $30 \mathrm{~s}$ apart), as observed on 11 July 2017 on the 7th Juno's close pass of the planet (north hemisphere); at this time, Ganymede was well south of the plasma sheet. The secondary, upstream spot could be a TEB spot, and the main spot-TEB distance of $1500 \mathrm{~km}$ is consistent with similar measurements in the far UV at the same longitude (28). However, the JIRAM images show each spot consists of two identical peaks $170 \mathrm{~km}$ apart. This separation corresponds to a tangential separation of $\sim 10000 \mathrm{~km}$ at the orbit of Ganymede, if magnetically mapped with a recent model (29) in a similar way as the Io footprint. Each spot is also followed by a very faint tail (26), which appears to be twisted.

As with the Io footprint, the spatial separations of features in the Ganymede footprint are not consistent with the simple specular reflection of Alfvén waves. In the Ganymede case, however, there is no trailing array of spots, with only two visible. A possible explanation is that both the leading and trailing magnetic reconnection regions in Ganymede magnetosphere [(34), their Fig. 1] generate locally enhanced Alfvén waves. If so, the separation between the peaks is related to the size of Ganymede's magnetosphere. Both the TEB and the main GFP display this double structure, perhaps suggesting that the cause is located at the origin of both the TEB and the main GFP Alfvén waves. However, other scenarios, such as reflections in the ionospheric resonator (35), could be invoked as well.

Auroral footprints of jovian moons are peculiar, but not unique, as a similar footprint of Enceladus is observed at Saturn (36), and even tentative evidence of footprints of extraso-lar planets on their central stars exists $(37,38)$. These auroral processes apply to any electrically-conducting moon orbiting within a planet's magnetosphere. The Juno/JIRAM high-resolution images of the satellite footprints in the IR show substructures, both in the spots and in the extended tail. 
The close-spaced, multiple spots require a process that occurs at smaller scales than would be produced by a single pair of Alfvén wings, in linear regime, bouncing between hemispheres, suggesting that they are produced by interactions occurring close to the moon, such as a train of vortices generated in Io's wake. Alternatively, interference between Alfvén waves, rather than reflections, could be responsible. Our images show that the extended tail can occasionally form two parallel arcs. Since this bifurcation is not observed often, it is possible that it is triggered by the northern anomaly in Jupiter's magnetic field. The Ganymede aurora expresses as twin spots, which may probe Ganymede's distant magnetosphere using the auroral emission.

\section{REFERENCES AND NOTES}

1. J. E. P. Connerney, R. Baron, T. Satoh, T. Owen, Images of excited H3+ at the foot of the Io Flux Tube in Jupiter's atmosphere. Science 262, 1035-1038 (1993). doi:10.1126/science.262.5136.1035 Medline

2. J. T. Clarke, G. E. Ballester, J. Trauger, R. Evans, J. E. P. Connerney, K. Stapelfeldt, D. Crisp, P. D. Feldman, C. J. Burrows, S. Casertano, J. S. Gallagher, R. E. Griffiths, J. J. Hester, J. G. Hoessel, J. A. Holtzman, J. E. Krist, V. Meadows, J. R. Mould, P. A. Scowen, A. M. Watson, J. A. Westphal, Far-ultraviolet imaging of Jupiter's Aurora and the Io "footprint". Science 274, 404-409 (1996). doi:10.1126/science 2745286404

3. D. J. Williams, B. H. Mauk, R. E. McEntire, E. C. Roelof, T. P. Armstrong, B. Wilken, J.G. Roederer, S. M. Krimigis, T. A. Fritz, L. J. Lanzerotti, Electron beams and ion composition measured at Io and in its torus. Science 274, 401-403 (1996). doi:10.1126/science.274.5286.401 Medline

4. D. J. Williams, R. M. Thorne, Energetic particles over Io's polar caps. J. Geophys. Res. 108 (A11), 1397 (2003). doi:10.1029/2003/A009980

5. L. A. Frank, W. R. Paterson, Intense electron beams observed at Io with the Galileo spacecraft. J. Geophys. Res. 104 (A12), 28657-28669 (1999). doi:10.1029/1999JA900402

6. P. Goldreich, D. Lynden-Bell, Io, a jovian unipolar inductor. Astrophys. J. 156, 59-78 (1969). doi:10.1086/149947

7. M. H. Acuha, F. M. Neubauer, N. F. Ness, Standing Alfven wave current system at Io: Voyager 1 observations. J. Geophys. Res. 86 (AIO), 8513-8521 (1981). doi-101029/JA086iA10p08513

8. F. M. Neubauer, Nonlinear standing Alfven wave current system at Io: Theory. J. Geophys. Res. 85 (A3), 1171-1178 (1980). doi:10.1029/JA085iA03p01171

9. J. E. P. Connerney, T. Satoh, The $\mathrm{H}^{+}+$ion: A remote diagnostic of the Jovian magnetosphere. Philos. Trans. R. Soc. London Ser. A 358, 2471-2483 (2000). doi:10.1098/rsta.2000.0661

10. F. Bagenal, Alfven wave propagation in the Io plasma torus. J. Geophys. Res. 88 (A4), 3013-3025 (1983). doi-101029/JA088iA04p03013

11. J. T. Clarke, J. Ajello, G. Ballester, L. Ben Jaffel, J. Connerney, J.-C. Gérard, G. R. Gladstone, D. Grodent, W. Pryor, J. Trauger, J. H. Waite Jr., Ultraviolet emissions from the magnetic footprints of Io, Ganymede and Europa on Jupiter. Nature 415, 997-1000 (2002). doi:10.1038/415997a Medline

12. J.-C. Gérard, A. Saglam, D. Grodent, J. T. Clarke, Morphology of the ultraviolet Io footprint emission and its control by Io's location. J. Geophys. Res. 111 (A4), A04202 (2006). doi:10.1029/2005JA011327

13. B. Bonfond, D. Grodent, J.-C. Gérard, A. Radioti, V. Dols, P. A. Delamere, J. T. Clarke, The Io UV footprint: Location, inter-spot distances and tail vertical extent. J. Geophys. Res. 114 (A7), A07224 (2009). doi:10.1029/2009JA014312

14. A. Adriani, G. Filacchione, T. Di lorio, D. Turrini, R. Noschese, A. Cicchetti, D. Grassi, A. Mura, G. Sindoni, M. Zambelli, G. Piccioni, M. T. Capria, F. Tosi, R. Orosei, B. M. Dinelli, M. L. Moriconi, E. Roncon, J. I. Lunine, H. N. Becker, A. Bini, A. Barbis, L. Calamai, C. Pasqui, S. Nencioni, M. Rossi, M. Lastri, R. Formaro, A. Olivieri, JIRAM, the Jovian Infrared Auroral Mapper. Space Sci. Rev. 213, 393-446 (2014). doi:10.1007/s11214014-0094-y 
15. S. J. Bolton, A. Adriani, V. Adumitroaie, M. Allison, J. Anderson, S. Atreya, J. Bloxham, S. Brown, J. E. P. Connerney, E. De Jong, W. Folkner, D. Gautier, D. Grassi, S. Gulkis, T. Guillot, C. Hansen, W. B. Hubbard, L. less, A. Ingersoll, M. Janssen, J. Jorgensen, Y. Kaspi, S. M. Levin, C. Li, J. Lunine, Y. Miguel, A. Mura, G. Orton, T. Owen, M. Ravine, E. Smith, P. Steffes, E. Stone, D. Stevenson, R. Thorne, J. Waite, D. Durante, R. W. Ebert, T. K. Greathouse, V. Hue, M. Parisi, J. R. Szalay, R. Wilson, Jupiter's interior and deep atmosphere: The initial pole-to-pole passes with the Juno spacecraft. Science 356, 821-825 (2017).

doi:10.1126/science.aal2108 Medline

16. F. Bagenal, A. Adriani, F. Allegrini, S. J. Bolton, B. Bonfond, E. J. Bunce, J. E. P. Connerney, S. W. H. Cowley, R. W. Ebert, G. R. Gladstone, C. J. Hansen, W. S. Kurth, S. M. Levin, B. H. Mauk, D. J. McComas, C. P. Paranicas, D. Santos-Costa, R. M. Thorne, P. Valek, J. H. Waite, P. Zarka, Magnetospheric science objectives of the Juno Mission. Space Sci. Rev. 213, 219-287 (2017). doi:10.1007/s11214-014-0036-8

17. J. E. P. Connerney, A. Adriani, F. Allegrini, F. Bagenal, S. J. Bolton, B. Bonfond, S. W. H. Cowley, J.-C. Gerard, G. R. Gladstone, D. Grodent, G. Hospodarsky, J. L. Jorgensen, W. S. Kurth, S. M. Levin, B. Mauk, D. J. McComas, A. Mura, C. Paranicas, E. J. Smith, R. M. Thorne, P. Valek, J. Waite, Jupiter's magnetosphere and aurorae observed by the Juno spacecraft during its first polar orbits. Science 356,826-832 (2017). doi:10.1126/science.aam5928 Medline

18. A. Mura, A. Adriani, F. Altieri, J. E. P. Connerney, S. J. Bolton, M. L. Moriconi, J.-C. Gerard, W. S. Kurth, B. M. Dinelli, F. Fabiano, F. Tosi, S. K. Atreya, F. Bagenal, G. R. Gladstone, C. Hansen, S. M. Levin, B. H. Mauk, D. J. McComas, G. Sindoni, G. Filacchione, A. Migliorini, D. Grassi, G. Piccioni, R. Noschese, A. Cicchetti, D. Turrini, S. Stefani, M. Amoroso, A. Olivieri, Infrared observations of Jovian aurora from Juno's first orbits: Main oval and satellite footprints. Geophys. Res. Lett. 44, 5308-5316 (2017). doi:10.1002/2017GL072954

19. T. Stallard, S. Miller, G. Millward, R. D. Joseph, On the dynamics of the Jovian ionosphere and thermosphere II: The measurement of $\mathrm{H}^{+}$vibrational 3 temperature, column density and total emission. Icarus 156, 498-514 (2002). doi:10.1006/icar.2001.6793

20. J. E. P. Connerney, M. H. Acuna, N. Ness, T. Satoh, New models of Jupiter's magnetic field constrained by the Io flux tube foot- print. J. Geophys. Res. 103 (A6), 11,929-11,939 (1998). doi:10.1029/97JA03726

21. S. L. G. Hess, P. A. Delamere, V. Dols, B. Bonfond, D. Swift, Power transmission and particle acceleration along the lo flux tube. J. Geophys. Res. 115 (A6), A06205 (2010). doi:10.1029/2009JA014928

22. J.-C. Gérard, A. Mura, B. Bonfond, G. R. Gladstone, A. Adriani, V. Hue, B. M. Dinelli, T. K. Greathouse, D. Grodent, F. Altieri, M. L. Moriconi, A. Radioti, J. E. P. Connerney, S. J. Bolton, S. M. Levin, Concurrent ultraviolet and infrared observations of the north Jovian aurora during Juno's first perijove. Icarus 312, 145-156 (2018). doi:10.1016/j.icarus.2018.04.020

23. S. Jacobsen, F. M. Neubauer, J. Saur, N. Schilling, Io's nonlinear MHD-wave field in the heterogeneous Jovian magnetosphere. Geophys. Res. Lett. 34, L10202 (2007). doi:101029/2006GL029187

24. J. Saur, F. M. Neubauer, D. F. Strobel, M. E. Summers, lo's ultraviolet aurora: Remote sensing of Io's interaction. Geophys. Res. Lett. 27, 2893-2896 (2000). doi:10.1029/2000GL003824

25. T. von Kármán, Aerodynamics (McGraw-Hill, 1963).

26. B. Bonfond, J. Saur, D. Grodent, S. V. Badman, D. Bisikalo, V. Shematovich, J.-C. Gérard, A. Radioti, The tails of the satellite auroral footprints at Jupiter. J. Geophys. Res. 122, 7985-7996 (2017). doi:10.1002/2017JA024370

27. C. Tao, S. V. Badman, M. Fujimoto, UV and IR auroral emission model for the outer planets: Jupiter and Saturn comparison. Icarus 213, 581-592 (2011). doi:10.1016/j.icarus.2011.04.001

28. B. Bonfond, S. Hess, F. Bagenal, J.-C. Gérard, D. Grodent, A. Radioti, J. Gustin, J. T. Clarke, The multiple spots of the Ganymede auroral footprint. Geophys. Res. Lett. 40, 4977-4981 (2013). doi:10.1002/grl.50989 
29. J. E. P. Connerney, S. Kotsiaros, R. J. Oliversen, J. R. Espley, J. L. Joergensen, P. S. Joergensen, J. M. G. Merayo, M. Herceg, J. Bloxham, K. M. Moore, S. J. Bolton, S. M. Levin, A new model of Jupiter's magnetic field from Juno's first nine orbits. Geophys. Res. Lett. 45, 2590-2596 (2018). doi:10.1002/2018GL077312

30. A. Adriani, A. Mura, M. L. Moriconi, B. M. Dinelli, F. Fabiano, F. Altieri, G. Sindoni, S. J. Bolton, J. E. P. Connerney, S. K. Atreya, F. Bagenal, J.-C. M. C. Gérard, G. Filacchione, F. Tosi, A. Migliorini, D. Grassi, G. Piccioni, R. Noschese, A. Cicchetti, G. R. Gladstone, C. Hansen, W. S. Kurth, S. M. Levin, B. H. Mauk, D. J. McComas, A. Olivieri, D. Turrini, S. Stefani, M. Amoroso, Preliminary JIRAM results from Juno polar observations: 2. Analysis of the Jupiter southern $\mathrm{H}_{3}{ }^{+}$emissions and comparison with the north aurora. Geophys. Res. Lett. 44, 4633-4640 (2017). doi:10.1002/2017GL072905

31. S. L. G. Hess, B. Bonfond, V. Chantry, J.-C. Gérard, D. Grodent, S. Jacobsen, A. Radioti, Evolution of the lo footprint brightness II: Modeling. Planet. Space Sci. 88, 76-85 (2013). doi:10.1016/j.pss.2013.08.005

32. Y. Su et al., Io-related Jovian auroral arcs: Modeling parallel electric fields. J. Geophys. Res. 108,1094 (2002).

33. D. Grodent, B. Bonfond, J.-C. Gérard, A. Radioti, J. Gustin, J. T. Clarke, J. Nichols, J. E. P. Connerney, Auroral evidence of a localized magnetic anomaly in Jupiter's northern hemisphere. J. Geophys. Res. 113 (A9), A09201 (2008). doi:10.1029/2008JA013185

34. M. G. Kivelson, J. Warnecke, L. Bennett, S. Joy, K. K. Khurana, J. A. Linker, C. T. Russell, R. J. Walker, C. Polanskey, Ganymede's magnetosphere: Magnetometer overview. J. Geophys. Res. 103 (E9), 19963-19972 (1998). doi:10.1029/98JE00227

35. Y. Su, S. T. Jones, R. E. Ergun, F. Bagenal, S. E. Parker, P. A. Delamere, R. L. Lysak, Io-Jupiter interaction: Alfve $\mathrm{n}$ wave propagation and ionospheric Alfvén resonator. J. Geophys. Res. 111 (A6), A06211 (2006). doi:10.1029/2005JA011252

36. W. R. Pryor, A. M. Rymer, D. G. Mitchell, T. W. Hill, D. T. Young, J. Saur, G. H. Jones, S. Jacobsen, S. W. H. Cowley, B. H. Mauk, A. J. Coates, J. Gustin, D. Grodent, J.-C. Gérard, L. Lamy, J. D. Nichols, S. M. Krimigis, L. W. Esposito, M. K. Dougherty, A. J. Jouchoux, A. I. F. Stewart, W. E. McClintock, G. M. Holsclaw, J. M. Ajello, J. E. Colwell, A. R. Hendrix, F. J. Crary, J. T. Clarke, X. Zhou, The auroral footprint of Enceladus on Saturn. Nature 472, 331-333 (2011). doi:10.1038/nature09928 Medline

37. E. Shkolnik, D. A. Bohlender, G. A. H. Walker, A. Collier Cameron, The on/off nature of star-planet interactions. Astrophys. J. 676, 628-638 (2008). doi:101086/527351

38. J. Saur, T. Grambusch, S. Duling, F. M. Neubauer, S. Simon, Magnetic energy fluxes in sub-Alfvénic planet star and moon planet interactions. Astron. Astrophys. 552, A119 (2013). doi:10.1051/0004-

$\underline{6361 / 201118179}$

\section{ACKNOWLEDGMENTS}

We thank ASI (Agenzia Spaziale Italiana) for support of the JIRAM contribution to the Juno mission.

Funding: JIRAM is funded with ASI (Agenzia Spaziale Italiana) contract number 2016- 353 23-H.O.B.B., D.G. and J.C.G. are supported by the PRODEX program managed by ESA in collaboration with the Belgian Federal Science Policy Office. T.K.G. is supported by the Juno Project under NASA grant NM06AAa75c to Southwest Research Institute. B.B. is funded by the Fund for Scientific Research (F.R.S.-FNRS).

Authors contributions: preparation of the manuscript, figures, calculations, data analysis: A.M., A.A.; Data interpretation: A.M., A.A., J.E.P.C, S.B., F.A., F.B., B.B., B.M.D., J.-C.G., T.G., D.G., S.L, B.M., M.L.M., J.S., J.H.W. Jr., D.T.; Revisions of the manuscript: A.M., A.A., J.E.P.C, S.B., F.A., F.B., B.B., B.M.D., J.-C.G., T.G., D.G., S.L, B.M., M.L.M., J.S., J.H.W. Jr.; Juno Principal investigator: S.B.; Responsible of JIRAM instrument: A.A.; Preparation for measurements, data acquisition: M.A., A.C., F.F., G.F., D.G., A.M., R.N., A.O., G.P., C.P., G.S., R.S., F.T., D.T.; Calibration: A.A., G.F., A.M, G.P.; Georeferencing: F.T., R.N., A.M.

Competing interests: The authors declare no competing interests.

Data and materials availability: Data in this study is publicly available on the Planetary Data System (http://pds.nasa.gov) and can be downloaded from http://atmos.nmsu.edu:8080/pds. The individual datasets are available at https://atmos.nmsu.edu/PDS/data/jnojir_Xxxx, where $x x x x$ is 1001,1002,1003 for EDR (Experiment Data Record - raw data) and 2001,2002, 2003 for RDR (Reduced Data Record - 
Published in : Science (2018), vol. 361, $n^{\circ} 6404$, pp. 774-777

DOI : $10.1126 /$ science.aat 1450

Status : Postprint (Author's version)

calibrated data) volumes. Noise reduction was performed using the algorithm in (18): a 2x1 pixel filter is applied to remove odd-even effect.

Fig. 1. Schematic illustration of the interaction between Io and the magnetosphere of Jupiter.

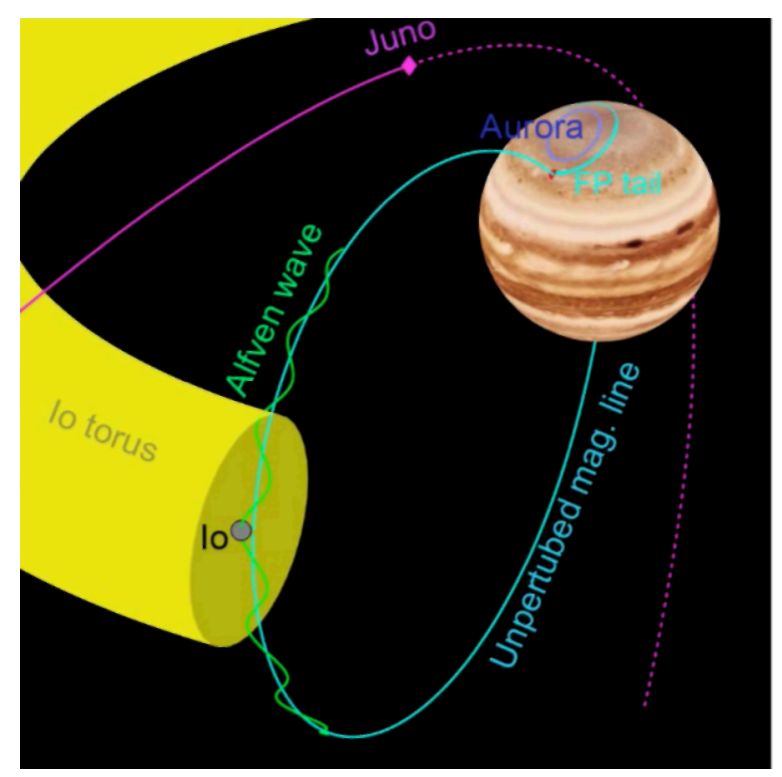

The figure shows Io, the Io plasma torus (yellow, simplified and not to scale), an unperturbed magnetic field line (cyan), an Alfvén wave (green, shape is simplified and not to scale), the main lo footprint (red), the footprint tail (cyan), the main auroral arc (blue), the trajectory and position of the Juno spacecraft. 

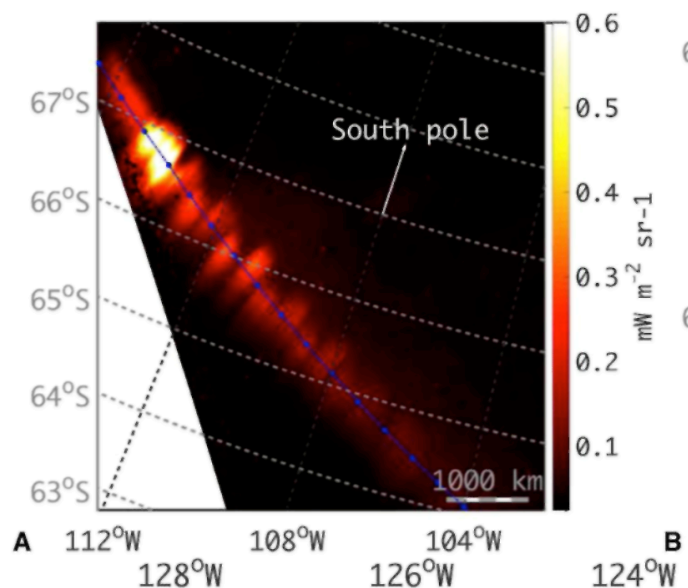

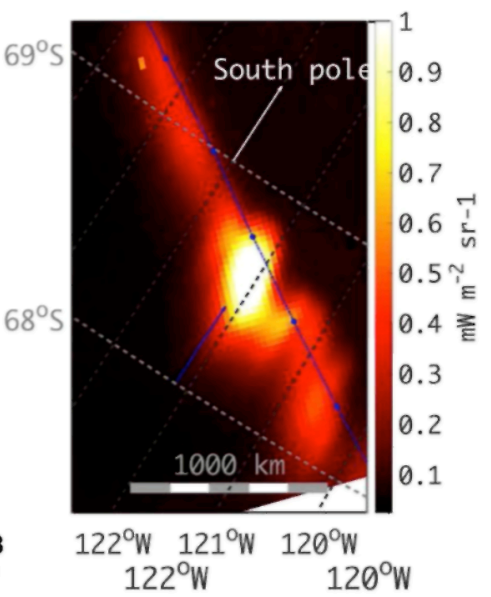

120

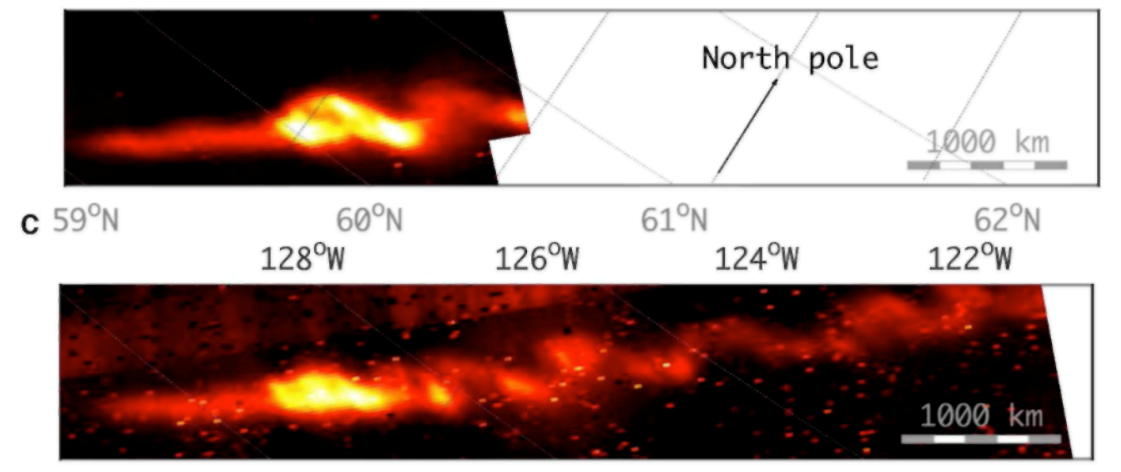

D

$59^{\circ} \mathrm{N}$

$60^{\circ} \mathrm{N}$

$61^{\circ} \mathrm{N}$

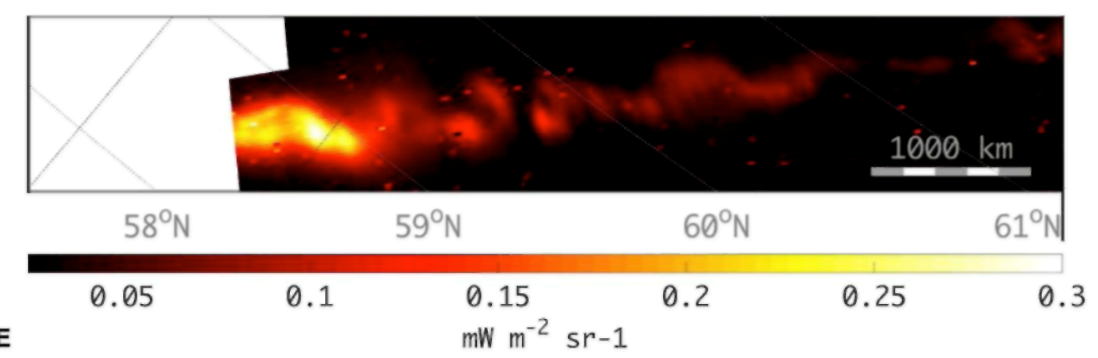

Fig. 2. All panels are shown in a polar orthographic projection with parallels and meridians overplotted.

(A) Footprint of Io on the south pole of Jupiter, as seen on 1 September 2017 at 22:50. The main spot (topleft corner of the image) is followed by a series of regularly spaced secondary spots, which are alternate in displacement above and below the median track (shown in blue). The thin blue arc with dots is the sequence of the predicted lo footprint positions (20), with dots showing its position every $100 \mathrm{~s}$. Radiances are in $\mathrm{mW} \mathrm{m}^{-2} \mathrm{sr}^{-1}$. (B) As in (A), but showing a later image taken at 23:09. The main spot (indicated by a blue arrow) has an oval shape, with an angular offset from the average direction of the tail (median track). (C to E) The Io footprint in the north aurora, observed at 20:43, 20:48 and 20:53 respectively on 1 September 2017. The model (20) is not shown in (C), (D), and (E) as it lies outside of the visible region. The black and white pixels [especially in (D)] are the effect of penetrating radiation affecting the detector. For a comparison with the results of Jacobsen et al.'s model, see Fig. 3 in (23). 
Fig. 3. The Io footprint tail, observed $\sim 100^{\circ}$ behind the main spot.

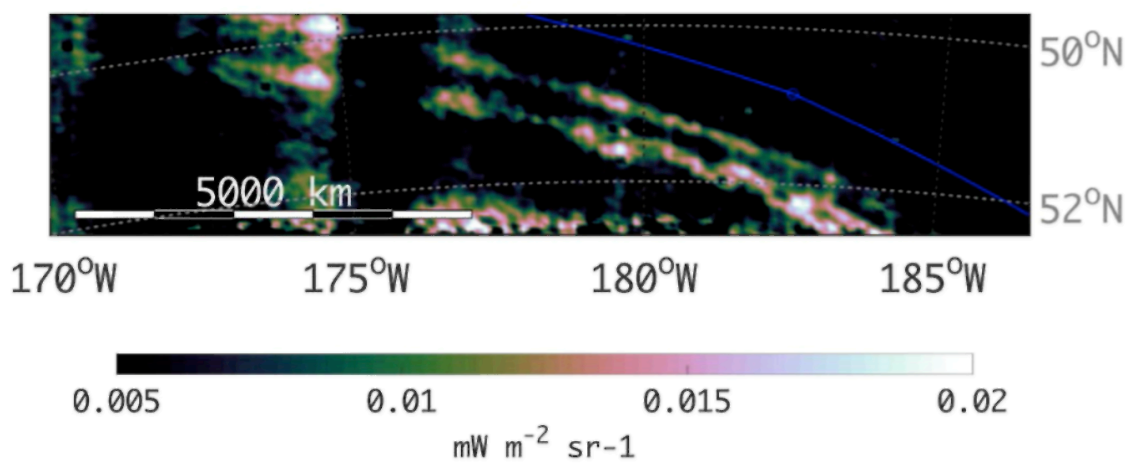

Image taken on 27 August 2016 (contour plot). The tail consists of two separate arcs. The red line is the prediction from a magnetic field model (20). The vertical artefacts on this image are produced by the noise-reduction routine. Local time is about dawn.

Fig. 4. Five consecutive JIRAM images of Ganymede footprints.
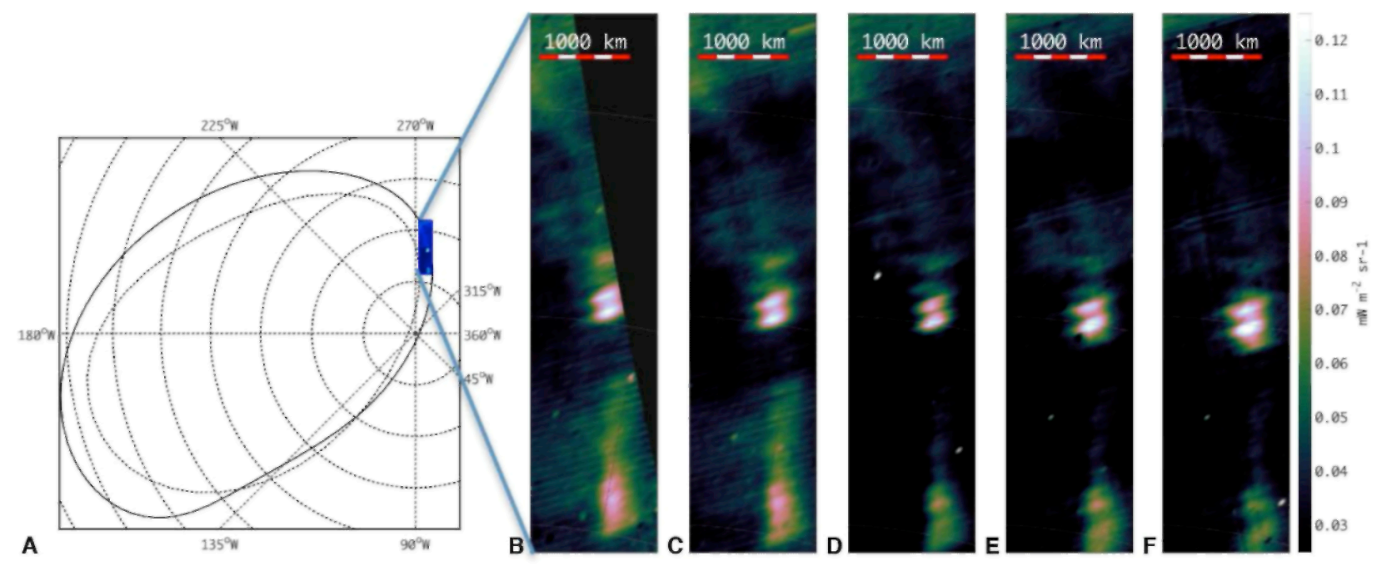

(A) diagram illustrating the main oval (dotted oval), Ganymede footprint (solid line) as predicted from recent magnetic field model (29) and the approximate location of the images in (B) to (F). (B to F) Images are taken from 00:07:45 to 00:09:48 on 11 July 2017 on the north hemisphere (contour plots). The main Ganymede footprint (in the middle of each image, and moving down) is preceded by a dimmer precursor spot. 